\title{
Qualidade de vida em adultos com Transtorno de Déficit de Atenção com Hiperatividade
}

\author{
Quality of life in adults with Attention Deficit Hyperactivity Disorder
}

\author{
Gustavo Luis Caribé Cerqueira ${ }^{1}$, Eduardo Pondé de Sena ${ }^{2 *}$
}

\begin{abstract}
${ }^{1}$ Mestrando em Processos Interativos de Órgãos e Sistemas pela Universidade Federal da Bahia (UFBA); ${ }^{2}$ Professor Associado de Farmacologia e Terapêutica do Departamento de Biorregulação do Instituto de Ciências da Saúde da
\end{abstract} UFBA.

\begin{abstract}
Resumo
Introdução: mais de 50\% das crianças com transtorno de déficit de atenção com hiperatividade (TDAH) continuam apresentando sintomas na vida adulta, com impactos no desenvolvimento psicossocial, profissional, acadêmico e emocional e, consequentemente, na qualidade de vida (QV). Objetivo: descrever características e escores da QV de dados da linha de base de uma amostra clínica de indivíduos adultos, participantes de ensaio clínico randomizado controlado do uso de estimulação transcraniana por corrente contínua no TDAH. Metodologia: sessenta indivíduos foram selecionados e submetidos à avaliação da QV com a escala de autorrelato de adultos (ASRS) e a escala da qualidade de vida em adultos com TDAH (AAQoL). Características demográficas e clínicas dos indivíduos foram descritas como frequências e porcentagens para variáveis categóricas. Resultados: a média (DP) do escore total de AAQoL foi de 43,0 (14,1). Os escores médios (DP) das subescalas foram 37,4 $(15,8)$ para Produtividade na Vida, 38,4 $(23,0)$ para Saúde Psicológica, 53,7 (15,5) para Perspectiva de Vida e 45,9 (22,2) para Relacionamentos. Conclusão: os resultados obtidos com o AAQoL demonstraram que os sujeitos da amostra caracterizaram o perfil do adulto portador de TDAH, onde diversas esferas da vida são comprometidas, em especial os "Relacionamentos", em que apresentou o maior comprometimento identificado, além da "Produtividade". Foi identificado também o consumo de bebida alcoólica e a hereditariedade com familiares também portadores, como confirma a literatura.
\end{abstract}

Palavras-chave: Qualidade de vida. Transtorno de déficit de atenção com hiperatividade. Adulto.

\begin{abstract}
Introduction: more than $50 \%$ of children with attention deficit / hyperactivity disorder (ADHD) continue to show symptoms in adulthood, with impacts on psychosocial, professional, academic, and emotional development and, consequently, on quality of life (QoL). Objective: to describe characteristics and QoL scores of baseline data from a clinical sample of adult subjects participating in a randomized controlled clinical trial of the use of transcranial by continuous stimulation in ADHD. Methodology: sixty individuals were selected and submitted to QoL assessment using the adult self-report scale (ASRS) and the quality of life scale in adults with $A D H D(A A Q o L)$. Demographic and clinical characteristics of individuals were described as frequencies and percentages for categorical variables. Results: the mean (SD) of the total AAQoL score was 43.0 (14.1). The mean scores (SD) of the subscales were 37.4 (15.8) for Productivity in Life, 38.4 (23.0) for Psychological Health, 53.7 (15.5) for Life Perspective and 45.9 (22.2) for Relationships. Conclusion: the results obtained with the AAQoL demonstrated that the subjects of the sample characterized the profile of the adult with ADHD where different spheres of life are compromised, especially the "Relationships" in which he presented the greatest impairment identified, in addition to "Productivity". The consumption of alcoholic beverages and heredity with family members also had been identified, as confirmed by the literature.

Key words: Quality of life. Attention deficit / hyperactivity disorder. Adult.
\end{abstract}

\section{INTRODUÇÃO}

O transtorno de déficit de atenção com hiperatividade (TDAH) é uma doença do neurodesenvolvimento caracterizada por sintomas centrais de desatenção, como distração e dificuldade de concentração, além de hiperatividade e impulsividade, como é caracterizada na quinta edição do Manual Diagnóstico e Estatístico de Transtornos Mentais

Correspondente/Corresponding: *Eduardo Pondé de Sena - Universidade Federal da Bahia, Instituto de Ciência e Saúde, Departamento de Biorregulação - End: Av. Reitor Miguel Calmon s/n, Vale do Canela, CEP: 40231-300 Salvador, BA - Tel: (71) 3283-8908 - E-mail: eduardoponde@hotmail.com
(DSM -5) (AMERICAN PSYCHIATRIC ASSOCIATION, 2013).

De acordo com Mattos et al. (2013) a forma adulta do transtorno do déficit de atenção com hiperatividade (TDAH) - anteriormente chamado de "tipo residual" - foi oficialmente reconhecida pela Associação Americana de Psiquiatria em 1980 na terceira edição do DSM, onde foi introduzida como uma condição clínica presente na vida adulta, embora até a quarta edição ainda fosse classificada como transtorno na infância. (SPITZER; MD; WILLIAMS, 1980).

Estudos epidemiológicos que abordam a ocorrência de TDAH em adultos ainda são escassos e mostram uma prevalência geral de 2,5\% a 3,4\% (FAYYAD et al., 2007; 
SIMON et al., 2009). Estima-se que mais de $50 \%$ das crianças com TDAH persistam com sintomas também na vida adulta (ARRUDA et al., 2015; MANNUZZA et al., 2011). Observa-se um declínio na impulsividade e hiperatividade com o avanço da idade, enquanto os sintomas de desatenção são bastante estáveis ao longo do tempo (BIEDERMAN; MICK; FARAONE, 2000). No entanto, da mesma forma do que crianças e adolescentes, os sintomas em indivíduos adultos com TDAH podem prejudicar gravemente o desempenho em contextos sociais, acadêmicos e profissionais, exercendo um impacto na qualidade de vida (QV) (VADALÀ et al., 2011).

O TDAH adulto está associado a muitos obstáculos na vida, como prejuízos nas realizações acadêmicas, ocupacionais e funcionais (ADLER et al., 2006; BIEDERMAN et al., 2008; BARKLEY; MURPHY; FISCHER, 2010; SOLANTO et al., 2008; STERN et al., 2017). Além disso, os indivíduos necessitam de intervenções visando melhorias nos problemas relacionados ao funcionamento emocional, familiar, social e interpessoal (KOOIJ et al., 2010). Não é de surpreender que pacientes adultos com TDAH apresentem menor QV quando comparados a indivíduos saudáveis, e que a gravidade dos sintomas de TDAH se correlacione negativamente com as medidas da QV (ADLER et al., 2006; BROD et al., 2015; MATTOS et al., 2013). Além disso, ensaios clínicos randomizados demonstraram algumas meIhorias nos parâmetros da qualidade de vida relacionados à resposta clínica a abordagens farmacológicas (ADLER et al., 2013; DURELL et al., 2013; GOTO et al., 2017) .

Aceitando a partir de diversas evidências cumulativas nos últimos anos os altos níveis de comprometimento funcional associado ao TDAH na fase adulta, tendendo a influenciar potencialmente de forma negativa na qualidade de vida. O objetivo do presente estudo foi descrever características e escores da QV de dados da linha de base de uma amostra clínica de indivíduos adultos, participantes de ensaio clínico randomizado controlado do uso de estimulação transcraniana por corrente contínua no TDAH. Além de descrever variáveis demográficas e psicossociais acerca dos impactos do TDAH em uma amostra clínica de adultos com TDAH.

\section{METODOLOGIA}

Para este ensaio clínico randomizado foi estimado um tamanho de amostra de 50 participantes com um poder de $80 \%$ para identificar uma diferença de $40 \%$ no desempenho de tarefa cognitiva entre os grupos, com uma probabilidade de erro tipo I de $0,05 \%$. Considerando a possibilidade de eventos inesperados, e considerando uma taxa de abandono de $20 \%$, a amostra final resultou em 60 indivíduos. O protocolo do ensaio controlado randomizado está registrado em clinicaltrials.gov (NCT01968512).

Setenta e três indivíduos com TDAH foram pré-selecionados, por entrevistas por e-mail e telefone, como possíveis participantes do estudo controlado randomizado. Posteriormente os sujeitos elegíveis foram selecionados em uma avaliação presencial no Instituto de Ciências da Saúde da Universidade Federal da Bahia (Salvador, Brasil), com o auxílio do Mini-International Neuropsychiatric Interview-Plus (MINI-Plus) e do Adult ADHD Self-Report Scale-18 (ASRS-18), conforme recomendado pela quarta edição revisada do Manual Diagnóstico e Estatístico de Transtornos Mentais (DSM-IV-TR). Um psiquiatra experiente também avaliou todos os indivíduos para confirmar o diagnóstico, (SHEEHAN et al., 1998) e o resultado do Adult ADHD Self-Report Scale-18 (ASRS-18), conforme recomendado pela quarta edição revisada do Manual Diagnóstico e Estatístico de Transtornos Mentais (DSM-IV-TR) (SHEEHAN et al., 1998; KESSLER et al., 2005).

Os critérios de inclusão foram: o diagnóstico de transtorno do déficit de atenção com hiperatividade com base no DSM-IV-TR, com 18 anos ou mais; e a capacidade de entender e assinar o termo de consentimento livre e esclarecido. Os critérios de exclusão foram: a presença de distúrbios psiquiátricos maiores, como esquizofrenia e transtorno bipolar; comprometimento cognitivo [pontuação $\leq 24$ no Mini Exame do Estado Mental (MEEM)]; analfabetismo; abuso de álcool e / ou uso de substâncias psicoativas nos 12 meses anteriores; e implantes metálicos no crânio ou dispositivo médico implantado (para fins de ensaios clínicos).

Todos os indivíduos selecionados para este estudo assinaram um termo de consentimento livre e esclarecido, após a explicação completa dos procedimentos do estudo, em conformidade com os princípios éticos para pesquisas médicas envolvendo seres humanos da Declaração de Helsinque (CARLSON; BOYD; WEBB, 2004). O Comitê de Ética em Pesquisa da Maternidade Climério de Oliveira da Universidade Federal da Bahia revisou e aprovou em nove de outubro de 2012 o protocolo de no 19311 (Número CAAE 07971612.2.0000.5543).

As seguintes variáveis foram descritas: sexo (feminino ou masculino); estado civil (solteiro, casado ou divorciado); renda (dependente, salário, aposentadoria ou não informada); atividade física (sim ou não); tratamento farmacológico (sim ou não); uso de psicoestimulantes (sim ou não); apresentação de psicoestimulantes (metilfenidato ou OROS metilfenidato); psicoterapia (sim ou não); comorbidade psiquiátrica (sim ou não); tipo de comorbidade psiquiátrica (transtorno depressivo, ansiedade generalizada, distúrbio, fobia social ou distúrbios combinados); gravidade do TDAH (leve, moderada ou grave); e forma de apresentação do TDAH (combinada, desatenta ou hiperativa / impulsiva).

A análise estatística foi realizada com o programa estatístico R Core Team (Versão 2020). A estatística descritiva foi utilizada para caracterizar a prevalência do impacto do TDAH em diferentes esferas da vida dos sujeitos da amostra. 


\section{RESULTADOS}

Setenta e três indivíduos foram inicialmente selecionados, 13 dos quais foram excluídos por não atenderem aos critérios de inclusão. A idade média (DP) da amostra foi $32,5(10,9)$ anos. A tabela 1 mostra a estatística descritiva para variáveis demográficas e clínicas.

Tabela 1 - Características demográficas e de TDAH

\begin{tabular}{lr}
\hline \multicolumn{1}{c}{ Características } & $\mathbf{n}=60$ \\
\hline Idade (média, DP) & $32,5 \pm 11,3$ \\
Gênero (n,\%) & \\
Masculino & $25(41,7 \%)$ \\
Feminino & $35(58,3 \%)$ \\
Subtipo TDAH (n,\%) & \\
Tipo Combinado & $32(53,3 \%)$ \\
Predominantemente Desatento & $18(30,0 \%)$ \\
Predominantemente Hiperativo/Impulsivo & $10(16,7 \%)$ \\
Escolaridade (n,\%) & \\
Possui apenas o Ensino Fundamental concluído & $3(5 \%)$ \\
Possui apenas o Ensino Médio concluído & $1(1,7 \%)$ \\
Possui o Ensino Superior incompleto & $32(53,3 \%)$ \\
Possui o Ensino Superior concluído & $24(40 \%)$ \\
Estado Civil (n,\%) & \\
Solteiro & $40(66,7 \%)$ \\
Casado & $17(28,3 \%)$ \\
Divorciado & $03(5 \%)$ \\
Comorbidade DSM-IV Eixo I (n,\%) & \\
Sim & $23(38,3 \%)$ \\
Não & $37(61,7 \%)$ \\
Tratamento farmacológico para TDAH & \\
Sim & $31(51,7 \%)$ \\
Não & $29(48,3 \%)$ \\
\hline${ }^{a}$ Idade expressa como média e desvio padrão (DP); ${ }^{b}$ According to criteria \\
of revised fourth edition of Diagnostic and Statistical Manual of Mental \\
Disorders (DSM-IV- $R$ ); ADHD, attention-deficit/hyperactivity disorder \\
Fonte: Dados da pesquisa &
\end{tabular}

O maior número de participantes da amostra foi do sexo feminino $58,3 \%(n=35)$ enquanto o público masculino abrangeu $41,7 \%(n=25)$. Mais de $90 \%$ da amostra relatou ter ingressado no ensino superior. Destes $38,3 \%$ $(n=23)$ concluíram pelo menos alguma faculdade. Acerca do estado civil, $66,7 \%(n=40)$ referiram ser solteiros(as), enquanto $28,3 \%(n=17)$ declararam ser casados(as) e $5 \%$ $(n=3)$ divorciadas(os).

A média (DP) do escore total de AAQoL foi de 42,9 $(13,9)$. Os escores médios (DP) das subescalas foram 37,4 $(15,8)$ para Produtividade na Vida, 38,4 (23,0) para Saúde Psicológica, 53,7 (15,5) para Perspectiva de Vida e 45,9 $(22,2)$ para Relacionamentos (tabela 2$)$.

Tabela 2 - Resultado total e subescalas AAQoL

\begin{tabular}{lcccc}
\hline & N & Média & Desvio Padrão & Escore \\
\hline Produtividade & 60 & 37,3 & 15,8 & 36,36 \\
Saúde psicológica & 60 & 38,4 & 23,0 & 45,83 \\
Perspectiva de vida & 60 & 53,7 & 15,5 & 53,57 \\
Relacionamentos & 60 & 45,9 & 22,2 & 15,00 \\
Total & 60 & 42,9 & 13,9 & 38,79 \\
\hline
\end{tabular}

Fonte: Dados da pesquisa.
O AAQoL é uma escala de autorrelato composta por 29 itens e foi desenvolvida para avaliar especificamente a QV relacionada à saúde de adultos com diagnóstico de TDAH durante as últimas duas semanas. Cada item é avaliado pelos pacientes em uma escala do tipo Likert de cinco pontos, variando de "nem um pouco / nunca" (1) a "extremamente / muito frequentemente" (5). O AAQoL produz uma pontuação total (com base em todos os 29 itens) e quatro pontuações de subescala: Produtividade de vida, Saúde psicológica, Perspectiva de vida e Relacionamentos. Pontuações totais e subescalas são calculadas: a) revertendo pontuações para todos os itens, exceto os sete itens na subescala Perspectiva de vida; b) transformando todas as pontuações de itens em uma escala de 0 a 100 pontos ( $1=0 ; 2=25 ; 3=50 ; 4=75 ; 5=100)$; e c) somando as pontuações dos itens e dividindo pela contagem dos itens para gerar a subescala e as pontuações totais. Pontuações mais altas no AAQoL indicam melhor funcionamento.

Referente ao escore total, todos os sujeitos da amostra apresentaram uma pontuação abaixo de 50\% $(38,79$ pontos) do escore total, o que indica um comprometimento global nas diversas esferas da vida desses indivíduos. A subescala "Relacionamentos" foi a escala que apresentou o maior comprometimento identificado (15 pontos), seguida da subescala "Produtividade" (36,36 pontos). As subescalas "Saúde psicológica" e "Perspectiva de vida" foram as que apresentaram os melhores escores, $(45,83$ e 53,57 respectivamente).

Acerca dos cuidados em saúde, no momento da avaliação $51,7 \%$ ( $n=31$ ) não faziam nenhum tipo de tratamento médico para o TDAH, enquanto $48,3 \%$ ( $n=$ 29) já realizavam tratamento médico específico para o TDAH (Tabela 3).

Tabela 3 - Cuidados em saúde

\begin{tabular}{ll}
\hline & $\mathbf{n}=60$ \\
\hline Acompanhamento psicológico ( $\mathrm{n}, \%)$ & $31(51,7 \%)$ \\
Faz psicoterapia & $29(48,3 \%)$ \\
Não faz psicoterapia & \\
Acompanhamento médico ( $\mathrm{n}, \%)$ & $29(49,3 \%)$ \\
Tem acompanhamento médico para o TDAH & $31(51,7 \%)$ \\
Não tem acompanhamento médico para o TDAH & \\
Atividades físicas ( $\mathrm{n}, \%)$ & $30(50 \%)$ \\
Desenvolve atividades físicas & $30(50 \%)$ \\
Não desenvolve atividades físicas & \\
\hline
\end{tabular}

Fonte: Dados da pesquisa.

Da amostra dos 60 sujeitos $51,7 \%(n=31)$ faziam psicoterapia, enquanto $48,3 \%$ ( $n=29$ ) não faziam. Sobre o período de início do acompanhamento psicológico, $15,2 \%(n=5)$ iniciaram até os 10 anos, enquanto $33,4 \%$ $(n=11)$ iniciaram o acompanhamento na faixa etária entre 11 e 25 anos. 27,3\% ( $n=9)$ iniciaram o acompanhamento psicológico entre 26 e 40 anos. 9,1\% $(n=3)$ iniciaram na faixa etária de 41 a 50 anos e $15 \%$ ( $n=5)$ iniciaram o acompanhamento depois dos 50 anos (Tabela 3). 
Uma questão importante refere-se à duração do tratamento, uma vez que a eficácia dos psicoestimulantes é reconhecida como sendo mais significativa nos primeiros seis meses de tratamento, com uma diminuição na resposta quando usada cronicamente (FINDLING, 2008; VOLKOW, SWANSON, 2013). Outra questão importante diz respeito à dose e adesão ao tratamento, uma vez que muitos indivíduos, devido à própria natureza do TDAH, fazem uso irregular dos psicoestimulantes, além de, eventualmente, tomarem doses abaixo do ideal. Além disso, em um seguimento mais duradouro, pode-se observar que, no mudo real, muitos pacientes adultos com TDAH descontinuam seu tratamento farmacológico (EDVINSSON; EKSELIUS, 2018).

Tabela 4 - Início do tratamento psicológico

\begin{tabular}{lr}
\hline \multicolumn{1}{c}{ Início do tratamento psicológico $(\mathbf{n}, \%)$} & \multicolumn{1}{c}{$\mathbf{n = 3 3}$} \\
\hline Até os 10 anos & $5(15,2 \%)$ \\
11 aos 25 anos & $11(33,0 \%)$ \\
26 aos 40 anos & $9(27,3 \%)$ \\
41 aos 50 anos & $3(9,1 \%)$ \\
Acima de 50 anos & $5(15,0 \%)$ \\
\hline
\end{tabular}

Fonte: Dados da pesquisa.

Entre os sujeitos da amostra 50\% ( $n=30)$ não possuíam familiar com sintomas ou diagnóstico de TDAH, enquanto $41,7 \%(n=25)$ possuíam familiares com diagnósticos de TDAH e 8,3\% ( $n=5)$ não sabiam informar sobre esta questão. Dos sujeitos que possuem familiares com diagnóstico de TDAH $36 \%$ ( $n=9$ ) possui mais de um membro na família com diagnóstico confirmado; $20 \%(n=5)$ relatou ter a mãe com diagnóstico de TDAH; $16 \%(n=4)$ ter os filhos/filhas com o diagnóstico; $12 \%(n=3)$ relatou ter primos/primas; $8 \%(n=2)$ têm o pai e $4 \%(n=1)$ relatou ter ou irmão/irmã ou sobrinhos/sobrinhas com o transtorno (Tabela 5).

Tabela 5 - Familiar e TDAH

\begin{tabular}{lr}
\hline & \multicolumn{1}{c}{$\mathbf{n = 2 5}$} \\
\hline Não (n,\%) & $30(50,0 \%)$ \\
Sim & $25(41,7 \%)$ \\
Não sabe informar & $5(8,3 \%)$ \\
& \\
Parentesco (n,\%) & \\
Pai & $2(8,0 \%)$ \\
Mãe & $5(20,0 \%)$ \\
Irmão/irmã & $1(4,0 \%)$ \\
Primos/primas & $3(12,0 \%)$ \\
Filhos/filhas & $4(16,0 \%)$ \\
Sobrinhos/sobrinhas & $1(4,0 \%)$ \\
Mais de um familiar & $9(36,0 \%)$ \\
\hline
\end{tabular}

Fonte: Dados da pesquisa.

Da amostra identificou-se que $66,7 \%(n=40)$ nunca se envolveram em acidentes enquanto $31,7 \%(n=19)$ têm em seu histórico acidentes. Dentro deste universo da amostra dos sujeitos que já sofreram acidentes: $31,6 \%$ $(n=6)$ sofreram acidentes de carro; $26,3 \%(n=5)$ sofreram alguma queda; $21,1 \%(n=4)$ foram atropelados; $10,5 \%$ $(n=2)$ praticaram agressão física; 5,3\% $(n=1)$ sofreram acidente de moto; e 5,3\% (n=1) praticaram algum crime relacionado a homicídio.

Tabela 6 - Envolvimento em acidentes

\begin{tabular}{lr}
\hline & $\mathrm{n}=\mathbf{6 0}$ \\
\hline Acidentes (n,\%) & \\
Não & $40(66,7 \%)$ \\
Sim & $19(31,7 \%)$ \\
& \\
Tipo de acidente (n,\%) & $\mathrm{n}=19$ \\
Moto & $1(5,3 \%)$ \\
Carro & $6(31,6 \%)$ \\
Agressão física & $2(10,5 \%)$ \\
Queda & $5(26,3 \%)$ \\
Homicídio & $1(5,3 \%)$ \\
Atropelamento & $4(21,1 \%)$ \\
\hline
\end{tabular}

Fonte: Dados da pesquisa.

Dos sujeitos que compuseram a amostra 58,3\% ( $n=35)$ fazem uso de bebida alcoólica e $41,7 \%(n=25)$ não. Destes que faziam uso de etílicos, $31,4 \%(n=11)$ o consumo datava de 6 a 10 anos; 28,6\% ( $n=10)$ entre 11 e 15 anos; $14,3 \%(n=5)$ faziam uso de bebida alcoólica entre um a cinco anos. O que chama mais atenção é uma parcela de $11,4 \%(n=4)$ que consumia bebida alcoólica há aproximadamente 30 anos. Entre o perfil de consumidor do tipo de bebida alcoólica mais consumida, 57,1\% $(n=20)$ ingeriam bebida fermentada, enquanto $14,3 \%(n=5)$ destilada e $28,6 \%(n=10)$ utilizavam tanto bebida destilada como fermentada.

Tabela 7 - Consumo de bebida alcoólica

\begin{tabular}{lr}
\hline & $\mathbf{n}=\mathbf{3 5}$ \\
\hline Tempo de consumo de bebida alcoólica $(\mathrm{n}, \%)$ & \\
entre 1 a 5 anos & $10(28,6 \%)$ \\
entre 6 a 10 anos & $11(31,4 \%)$ \\
entre 11 e 15 anos & $10(28,6 \%)$ \\
aproximadamente 30 anos & $4(11,4 \%)$ \\
Tipos de bebidas & \\
Destilada & $5(14,3 \%)$ \\
Fermentada & $20(57,1 \%)$ \\
Destilada e fermentada & $10(28,6 \%)$ \\
\hline
\end{tabular}

Fonte: Dados da pesquisa.

\section{DISCUSSÃO}

Existem limitações em nosso estudo. Os dados foram obtidos apenas uma vez na linha de base (baseline) e os pacientes foram avaliados em um único dia, tendo sido feitas as avaliações clínicas e escalas, sendo posteriormente submetidos à estimulação transcraniana por corrente 
contínua (ETCC), ativa ou simulada, em uma única sessão. Ademais, os dados unicamente da linha de base, transversais, impediram-nos de fazer inferências sobre causalidade ou fatores preditores. Um desenho do tipo corte transversal quando muito pode examinar associações, jamais causalidade. Como regra geral são necessários pelo menos 10 casos para cada variável independente em um modelo de regressão linear, para obter os melhores estimadores eficientes e imparciais não lineares (PEDUZZI et al., 1996). Com apenas 60 casos incluídos, não se poderia fazer análise de regressão linear múltipla.

Em estudo desenvolvido por Mitchell e outros (2017), foram testados os efeitos da participação de adultos com TDAH no MAP (Mindful Awareness Program) onde foram avaliados os sintomas do TDAH e seus impactos na vida destes adultos, em especial nas funções executivas e na desregulação emocional. Para isso os adultos foram estratificados por tipos de medicação para TDAH e randomizados em grupos para tratamento baseado em mindfulness e grupo de lista de espera. Os pesquisadores observaram uma melhora dos pacientes dos questionários de autorrelato e nas avaliações clínicas relacionadas aos sintomas da TDAH, tal como foi observado na regulação emocional e nas funções executivas.

Em estudo semelhante, Cole e outros (2016) direcionaram um treinamento baseado na Terapia Cognitivo Comportamental (TCC) ou DBT. Eles avaliaram os benefícios do programa de redução de sintomas residuais em pacientes com TDAH que apresentavam baixa resposta aos medicamentos. Nesse estudo, os pacientes participaram durante um ano do programa e receberam psicoterapia individual associada com terapia de grupo associadas com diferentes módulos, entre eles, mindfulness. $\mathrm{O}$ foco no tratamento era desenvolver habilidade como regulação emocional, eficácia interpessoal e tolerância ao estresse, além de maior controle da impulsividade/hiperatividade e atenção. No geral, o tratamento associado apresentou resultados bem significativos de melhora no desenvolvimento de habilidades mais adaptativas em todas as dimensões da vida dos pacientes.

Esses estudos reforçam os dados encontrados nesta pesquisa, que a psicoterapia é um importante recurso no tratamento do TDAH adulto e quanto mais cedo seu início, maior o desenvolvimento de habilidades para lidar com as suas comorbidades. Apesar de apenas $55 \%(n=33)$ realizarem acompanhamento psicológico, o que reflete também acompanhamento médico específico para o tratamento do TDAH, cerca de $49,3 \%(n=29)$. O que chama atenção para o tratamento combinado ser utilizado por aproximadamente $50 \%$ da amostra, explicando o baixo índice global no resultado do AAQoL.

Embora estudos interessantes tenham sido publicados avaliando a qualidade de vida de indivíduos em uso de drogas específicas (BRAMS et al., 2011; MATTOS et al., 2013; WEHMEIER et al., 2011;), é necessária e relevante investigação adicional comparando as diferentes terapias disponíveis, doses e suas implicações nos parâmetros da qualidade de vida, para estabelecer a melhor abordagem farmacológica. Esse tópico amplamente abordado em estudos sobre o TDAH é outra questão importante que pode afetar a qualidade de vida, principalmente eventos adversos como insônia, ansiedade e irritabilidade (ANTSHEL et al., 2011; BERMAN et al., 2009; FELDMAN; REIFF, 2014; OKIE, 2006; VOLKOW; SWANSON, 2013;).

Uma revisão sistemática recente analisou a qualidade de vida e os resultados funcionais em estudos de tratamento farmacológico para o TDAH em crianças, adolescentes e adultos (COGHILL et al., 2017). Em relação à população adulta, os autores encontraram efeitos significativos de agentes farmacológicos nos sintomas, qualidade de vida e funcionamento do TDAH. Tamanhos de efeito maiores foram observados para fármacos estimulantes do que para medicamentos não estimulantes; para crianças / adolescentes do que para adultos; e para sintomas do que para a qualidade de vida, revelando menores efeitos do tratamento farmacológico na modulação dos parâmetros da QV.

Em um estudo com 211 pacientes ambulatoriais adultos com TDAH, escores de desatenção e hiperatividade, sexo feminino, menor nível socioeconômico e transtornos de humor comórbidos foram associados a eventos negativos da vida. Possíveis fatores de confusão foram investigados por meio de modelos de regressão de Poisson e o efeito da gravidade da desatenção e hiperatividade nos eventos negativos da vida foi confirmado. Garcia et al. (2012) concluíram que eventos negativos da vida vivenciados por pacientes com TDAH estavam associados à gravidade dos sintomas, independentemente de transtornos psiquiátricos comórbidos.

Pacientes com sintomas de TDAH mais graves, determinados com base no escore da ASRS, apresentaram escores totais da AAQoL e suas subescalas significativamente menores (BROD et al., 2006; GARCIA et al., 2012). A ASRS é composta por uma seção com nove perguntas abordando sintomas de desatenção e outra seção com nove perguntas abordando manifestações de hiperatividade e impulsividade (KESSLER et al., 2005). Portanto, um escore mais alto do ASRS indica um número maior de sintomas, sugerindo maior comprometimento funcional de importantes domínios cognitivos, principalmente atenção e controle inibitório. Esses domínios são essenciais para um melhor planejamento e execução das atividades da vida diária e na interação do indivíduo com o meio ambiente, aspectos importantes associados à qualidade de vida. Ao mesmo tempo, pior qualidade de vida, menos motivação e menos satisfação contribuem para um maior comprometimento do desempenho do indivíduo em diferentes áreas, e qual seria a consequência pode se tornar uma causa, agravando o distúrbio. Portanto, o entendimento de que indivíduos com formas mais graves de TDAH apresentam maior comprometimento da QV, embora evidente, enfatiza a importância de uma terapia mais eficaz, com melhor controle dos sintomas e interferindo na qualidade de vida em uma abordagem mais ampla. 
A comorbidade psiquiátrica é frequente entre indivíduos com TDAH e frequentemente associada a um maior comprometimento funcional (MCGOUGH et al., 2005). De acordo com esses achados anteriores, maiores escores da QV foram encontrados entre os indivíduos sem comorbidade psiquiátrica, mas essa diferença não atingiu significância estatística $(p=0,07)$. O provável aumento no número e duração das terapias farmacológicas, abordagem de tratamento combinado e maior dificuldade no controle dos sintomas podem explicar a redução na QV entre indivíduos com comorbidade psiquiátrica em comparação com aqueles com TDAH isoladamente. Além disso, a sobreposição de sintomas pode ter um efeito aditivo ou mesmo sinérgico, agravando o prejuízo causado pelo TDAH e seu impacto na QV. A investigação dessas questões em uma amostra maior pode revelar diferenças estatisticamente significativas nos escores do AAQoL entre indivíduos com e sem comorbidade psiquiátrica.

Além das limitações mencionadas, nosso estudo apresenta outra limitação que deve ser considerada - a falta de um grupo controle. Um controle serviria como uma comparação com relação a aspectos demográficos importantes, como estado civil e renda, e permitiria uma análise mais detalhada dos domínios frequentemente prejudicados em indivíduos com TDAH, como relacionamentos e produtividade (BROD et al., 2005; BROD et al., 2006).

Uma consideração final diz respeito à noção da QV em indivíduos com TDAH. O conceito da QV é complexo e influenciado por aspectos culturais e pessoais (AGARWAL et al., 2012; BROD et al., 2006). No entanto, o comprometimento funcional decorrente dos sintomas do TDAH afeta a capacidade de avaliar adequadamente 0 ambiente circundante e as situações diárias. Isso aumenta o potencial dos sintomas de desatenção, hiperatividade e impulsividade para impactar na qualidade de vida, ressaltando a importância de avaliar esse aspecto em indivíduos com TDAH, independentemente das diferenças socioculturais.

De acordo com Franke, Neale e Faraone (2009), cinco estudos de associação genômica ampla (GWAS, na sigla em inglês) foram realizados no diagnóstico de TDAH e fenótipos relacionados. Quatro deles são baseados em um conjunto de amostra de 958 pais - trios de crianças coletados como parte do estudo International Multicentre ADHD Genetics (IMAGE) e genotipados com fundos da Genetic Association Information Network (GAIN).

Em estudo desenvolvido por Toshinobu et al. (2010) buscavam entender melhor a transmissão familiar do transtorno de déficit de atenção com hiperatividade (TDAH), tendo em vista este como um transtorno altamente hereditário e seus efeitos do estado de TDAH paterno e materno nos sintomas e subtipos de TDAH dos filhos. Os resultados denunciaram que os critérios de TDAH foram endossados por $23 \%$ dos pais e $27 \%$ das mães e, por pelo menos um dos pais, em $41 \%$ dos casos. A gravidade do TDAH foi maior para crianças cujos pais tinham TDAH do que para aquelas cujos pais não tinham. OTDAH paterno foi associado a um aumento da probabilidade do subtipo combinado de TDAH e uma diminuição da probabilidade do subtipo desatento em crianças do sexo masculino.

Esses estudos corroboram com os dados encontrados nesta pesquisa, em que $41 \%(n=25)$ possuíam alguém com grau de parentesco portador de TDAH. Destes $36 \%$ (n =9) possuíam mais de um familiar portador do transtorno. De acordo com o estudo de Toshinobu et al. (2010), a condição do TDAH dos pais parece conferir riscos diferentes para a gravidade dos sintomas de hiperatividade-impulsividade e desatenção, dependendo do sexo dos pais. O TDAH biparental não parece ter um efeito aditivo ou sinérgico sobre a gravidade do TDAH dos filhos.

Para Todd (2000), o risco da recorrência do TDAH entre pais e irmãos é cerca de cinco vezes maior do que na população geral. A agregação familiar compartilha uma proporção maior de informações genéticas e exposições ambientais do que os indivíduos escolhidos ao acaso na população. Quanto mais próximo o parentesco entre dois indivíduos de uma família, mais alelos eles têm em comum, herdados dos seus antepassados. O que aumenta a probabilidade de manifestação da doença (CIRCUNVIS, et al., 2017).

As evidências atuais sugerem que grande parte dos indivíduos afetados com TDAH permanece com a doença durante a vida adulta, resultando em problemas profissionais e sociais, bem como em risco aumentado para o desenvolvimento de outros transtornos mentais. Em estudo realizado por Arias e outros (2008), em que foram entrevistados 1.761 adultos com diagnóstico de uso abusivo de cocaína e / ou opioides ao longo da vida, o TDAH foi associado a uma idade mais precoce do uso da primeira substância, a diagnósticos psiquiátricos, a maior probabilidade de tentativa de suicídio e a maior número de hospitalizações. Corroborando os resultados acima, a nossa amostra demonstrou que 58,3\% $(n=35)$ dos indivíduos faziam uso de bebida alcoólica, com períodos de consumo mais longevos, entre dez e trinta anos.

Em relação aos $31,6 \%$ da nossa amostra que já haviam sofrido acidentes de carro, estudos como o de Barkley et al. (2002) corroboram com esse dado e também avaliaram a desatenção e a resposta impulsiva utilizando o Conners CPT de indivíduos diagnosticados com TDAH de acordo com os critérios do DSM-IV e um grupo controle de pessoas sem diagnóstico de TDAH. Esses escores geraram um tempo de reação, discriminação visual e inibição de resposta a estímulos visuais e o Inventário de Condução Comportamental Cognitivo de Autorrelato (CBDI). Apesar de não ter havido diferença entre os grupos na resposta impulsiva, o grupo com TDAH foi significativamente menos atento ao CPT. No CBDI, não foram encontradas diferenças entre os grupos de TDAH e controle no tempo de reação visual regular e tarefas de discriminação. Entretanto, o grupo com TDAH cometeu mais erros em tarefas de resposta diferencial de reação visual reversa, sugerindo taxas mais baixas de comportamento governado por regras e em uma tarefa de varredura visual, particularmente no campo visual direito. 
Quanto aos $26,3 \%$ dos sujeitos que sofreram alguma queda, estudos como de Shilon (2012) e Ertan, Özcan e Pepele (2012), Leibson et al. (2006) reconhecem que crianças e adolescentes com diagnóstico de TDAH são mais atendidas em serviços de emergência repetidamente em consequência de traumas. Estudos como de Sabuncuoglu, Taser e Berkem (2007) e Thikkurissy, McTigue e Coury (2012) demonstraram uma relação entre sintomas de hiperatividade e impulsividade com traumas dentários.

Além disso, $10,5 \%$ relataram terem praticado algum tipo de agressão física, dado reforçado pelos estudos de Barkley e outros (1990), Whalen e outros (2002), em que constatou-se que indivíduos com TDAH são mais propensos a exibir correlações de traços agressivos, como comportamento antissocial. Estudos adicionais relataram raiva e problemas com a raiva no controle em adultos e adolescentes com TDAH (RAMIREZ et al., 1997). Esses estudos sugerem que os indivíduos com TDAH podem ser mais propensos a dirigir agressivamente, o que pode ser um fator que contribui para o aumento do risco de dirigir. Esses dados corroboram com os 5,3\% que sofreram acidente de moto e os 5,3\% que praticaram algum crime relacionado a homicídio.

\section{CONCLUSÃO}

Apesar do pequeno número da amostra, estes achados corroboram com os resultados de outros estudos onde o TDAH adulto gera um impacto na qualidade de vida de seus portadores. Tais impactos interferem em suas rotinas, nos relacionamentos, no trabalho e no desempenho acadêmico. Deve-se atentar até mesmo como um fator de risco para acidentes consigo mesmo ou com terceiros. Em se tratando do consumo de bebida alcoólica, estudos têm evidenciado que pessoas com TDAH apresentam maior tendência ao consumo abusivo de álcool e outras drogas. Mesmo com uma amostra pequena, o consumo de álcool ficou configurado em mais de $50 \%$ da amostra. Enfim, mesmo com limitações, este estudo caracterizou a população adulta portadora de TDAH correspondendo ao que se compreende hoje no perfil destes indivíduos.

\section{REFERÊNCIAS}

ADLER, L. A. et al. Quality of life assessment in adult patients with attention-deficit/hyperactivity disorder treated with atomoxetine. Journal of Clinical Psychopharmacology, [s. I.], v. 26, n. 6, p. 648-652, Dec. 2006. Disponível em: https://journals.Iww.com/psychopharmacology/ Abstract/2006/12000/Quality_of_Life_Assessment_in_Adult_Patients_ With.16.aspx. Acesso em: 23 out. 2020

ADLER, L. A. et al. Self-Reported quality of life in adults with attentiondeficit/hyperactivity disorder and executive function impairment treated with lisdexamfetamine dimesylate: a randomized, double-blind, multicenter, placebo-controlled, parallel-group study. Bmc Psychiatry, [s. I.], v. 13, Oct 2013. Disponível em: https://pubmed.ncbi.nlm.nih. gov/24106804/. Acesso: 30 set. 2020.

AGARWAL, R. et al. The quality of life of adults with attention deficit hyperactivity disorder: a systematic review. Innovations in Clinical Neuroscience, [s. I.], v. 9, n. 5-6, p. 10-21, May, 2012. Disponível em:
https://www.ncbi.nlm.nih.gov/pmc/articles/PMC3398685/. Acesso em: 16 out. 2020.

AHNEMARK, E. et al. Health-related quality of life and burden of illness in adults with newly diagnosed attention-deficit/hyperactivity disorder in Sweden. BMC Psychiatry, [s. I.], v. 18, n. 1, p. 223, Jul. 2018. Disponível em: https://bmcpsychiatry.biomedcentral.com/articles/10.1186/ s12888-018-1803-y. Acesso em:

AMERICAN PSYCHIATRIC ASSOCIATION. Diagnostic and statistical manual of mental disorders. 5th ed. Washington, DC: American Psychiatric Association, 2013.

ANTSHEL, K. M. et al. Advances in understanding and treating ADHD. BMC Medicine, [s. I.], v. 9, n. 72, 2011.

Arias AJ, et al. Correlates of co-occurring ADHD in drug-dependent subjects: prevalence and features of substance dependence and psychiatric disorders. Addict Behav., [s. I.], v.33, n.9, p. 1199-1207, Sept 2008. Disponível em: https://pubmed.ncbi.nlm.nih.gov/18558465/. Acesso em: 20 out. 2020.

ARRUDA, M. A. et al. ADHD and mental health status in brazilian schoolage children. Journal of Attention Disorders, [s. I.], v. 19, n. 1, p. 11-7, Jan. 2015. Disponível em: https://pubmed.ncbi.nlm.nih.gov/22665924/. Acesso em: 16 out. 2020.

BARKLEY, R. A. et al. Driving in young adults with attention-deficit/ hyperactivity disorder: knowledge, performance, adverse outcomes, and the role of executive functioning. Journal of the International Neuropsychological Society, [s. I.], v. 8, n. 5, p. 655-672, Nov. 2002. Disponível em: https://www.cambridge.org/core/journals/journal-ofthe-international-neuropsychological-society/article/abs/driving-inyoung-adults-with-attention-deficit-hyperactivity-disorder-knowledgeperformance-adverse-outcomes-and-the-role-of-executive-functioning/ 175202B2BF8C1B1285CA90E424727258. Acesso em: 3 ago. 2020.

BARKLEY, R. A. et al. The adolescent outcome of hyperactive children diagnosed by research criteria: I. An 8-year prospective follow-up study. Journal of the American Academy of Child and Adolescent Psychiatry, [s. I.], v. 29, n. 4, p. 546-57, Jul. 1990. Disponível em: https://pubmed. ncbi.nlm.nih.gov/2387789/. Acesso em: 10 set. 2020.

BARKLEY, R. A.; MURPHY, K. R.; FISCHER, M. ADHD in adults: what the science says. New York: The Guilford Press, 2010.

BERMAN, S. M. et al. Potential adverse effects of amphetamine treatment on brain and behavior: a review. Mollecular Psychiatry, v. 14, n. 2, p. 123-42, Feb. 2009. Disponível em: https://www.nature.com/ articles/mp200890. Acesso em: 10 set. 2020.

BIEDERMAN, J. et al. High risk for attention-deficit/hyperactivity disorder among children of parents with childhood onset of the disorder: a pilot study. American Journal of Psychiatry, [s. I.], v. 152, n. 3, p. 431-5, Mar. 1995. Disponível em: https://pubmed.ncbi.nlm.nih.gov/7864271/. Acesso em: 10 set. 2020.

BIEDERMAN, J.; MICK, E.; FARAONE, S. V. Age-dependent decline of symptoms of attention deficit hyperactivity disorder: impact of remission definition and symptom type. American Journal of Psychiatry, [s. I.], v. 157, n. 5, p. 816-818, 2000. Acesso em: 10 set. 2020.

BIEDERMAN, J. et al. Educational and occupational underattainment in adults with attention-deficit/hyperactivity disorder: a controlled study. The Journal of clinical psychiatry, 2008. Disponível em: https://pubmed. ncbi.nlm.nih.gov/18681752/. Acesso: 10 set. 2020.

BRAMS, M. et al. Effects of open-label lisdexamfetamine dimesylate on self-reported quality of life in adults with ADHD. Postgrad Med. [s. I.] v. 123, n. 3, p. 99-108, May, 2011. Disponível em: https://pubmed.ncbi. nlm.nih.gov/21566420/. Acesso em: 15 set. 2020. 
BROD, M. et al. Validation of the adult attention-deficit/hyperactivity disorder quality-of-life scale in European patients: comparison with patients from the USA. ADHD Attention Deficit and Hyperactivity Disorders, [s. I.], n. 7, p. 141-150, June, 2015. Disponível em: https:// link.springer.com/article/10.1007/s12402-014-0160-z\#article-info. Acesso em: 12 jun. 2020.

BROD, M. et al. Validation of the adult attention-deficit/hyperactivity disorder quality-of-life Scale (AAQoL): a disease-specific quality-of-life measure. Quality of Life Research, [s. I.], v. 15, n. 1, p. 117-29, Feb. 2006. Disponível em: https://pubmed.ncbi.nlm.nih.gov/16411036/. Acesso em: 17 jul. 2020.

BROD, M. P. et al. Conceptualization and assessment of quality of life for adults with Attention Deficit Disorder. Primary Psychiatry, [s. I.], v. 12, n. 6, p. 58-64, 2005. Disponível em: https://www.researchgate. net/profile/Meryl_Brod/publication/232471361_Conceptualization_ and_Assessment_of_Quality_of_Life_for_Adults_with_AttentionDeficitHyperactivity_Disorder/links/53d26cf00cf220632f3c9993/ Conceptualization-and-Assessment-of-Quality-of-Life-for-Adults-withAttention-Deficit-Hyperactivity-Disorder.pdf. Acesso em: 01 set. 2020.

CARLSON, R. V.; BOYD, K. M.; WEBB, D. J. The revision of the Declaration of Helsinki: past, present and future. British Joural of Clinical Pharmacology, [s. I.], v. 57, n. 6, p. 695-713, Jun, 2004. Disponível em: https://www.ncbi.nlm.nih.gov/pmc/articles/PMC1884510/. Acesso em: 27 ot. 2020.

CANTWELL, D. P. Attention deficit disorder: a review of the past 10 years. Journal of the American Acaddemy of Child \& Adolescent Psychiatry, [s. I. ], v. 35, n. 8, p. 978-87, Aug. 1996. Disponível em: https://www. sciencedirect.com/science/article/abs/pii/S0890856709624916. Acesso em: 07 nov. 2020.

CIRCUNVIS, B. C. et al. Componentes bióticos e genéticos relacionados ao TDAH: revisão de literatura. Biosaúde, Londrina, v. 19, n. 2, 2017. Disponível em: http://www.uel.br/revistas/uel/index.php/biosaude/ article/view/31056. Acesso em: 21 out. 2020.

COGHILL, D. R. et al. Systematic review of quality of life and functional outcomes in randomized placebo-controlled studies of medications for attention-deficit/hyperactivity disorder. European Child \& Adolescent Psychiatry, Apr. 2017. Disponível em: https://pubmed.ncbi.nlm.nih. gov/28429134/. Acesso em: 04 ago. 2020.

COLE, P. et al. CBT/DBT skills training for adults with attention deficit hyperactivity disorder (ADHD). Psychiatria Danubina, [s. I.], n. 28, p. 103-107, 2016. Suppl 1. Disponível em: https://pubmed.ncbi.nlm.nih. gov/27663817/. Acesso em: 03 out. 2020.

COMINGS, D. E. et al. Multivariate analysis of associations of 42 genes in ADHD, ODD and conduct disorder. Clinical Genetics, [s. I.], v. 58, n. 1, p. 31-40, July, 2000. Disponível em: https://onlinelibrary.wiley.com/ doi/epdf/10.1034/j.1399-0004.2000.580106.x. Acesso em:10 jun. 2020.

COUTO, T. S.; MELO-JUNIOR, M. R.; GOMES, C. R. A. Aspectos neurobiológicos do transtorno do déficit de atenção e hiperatividade (TDAH): uma revisão. Ciência e Cognição, Rio de Janeiro, v. 15, n. 1, p. 241-251, 2010. Disponível em: http://www.cienciasecognicao.org/ revista/index.php/cec/article/view/202. Acesso em: 25 jun. 2020.

DURELL, T. M. et al. Atomoxetine treatment of attention-deficit/ hyperactivity disorder in young adults with assessment of functional outcomes: a randomized, double-blind, placebo-controlled clinical trial. Journal of Clinical Psychopharmacology, [s. I.], v. 33, n. 1, p. 45-54, Feb. 2013. Disponível em: https://journals.Iww.com/psychopharmacology/ Abstract/2013/02000/Atomoxetine_Treatment_of.9.aspx. Acesso em: 21 out. 2020.

EDVINSSON, D.; EKSELIUS, L. Long-term tolerability and safety of pharmacological treatment of adult Attention-Deficit/Hyperactivity Disorder: a 6-year prospective naturalistic study. Journal of Clinical
Psychopharmacology, [s. I.], v. 38, n. 4, p. 370-375, Aug. 2018. Disponível em: https://journals.Iww.com/psychopharmacology/ Fulltext/2018/08000/Long_Term_Tolerability_and_Safety_of.16.aspx. Acesso em: 08 ago. 2020.

ERTAN, C.; ÖZCAN, Ö. Ö.; PEPELE, M. S. Paediatric trauma patients and attention deficit hyperactivity disorder: correlation and significance. Emergency Medicine Journal, [s. I.], v. 29, n. 11, p. 911-4, 2012. Disponível em: https://pubmed.ncbi.nlm.nih.gov/22215173/. Acesso em: 02 nov. 2020.

FARAONE, S. V.; BIEDERMAN, J.; MICK, E. The age-dependent decline of attention deficit hyperactivity disorder: a meta-analysis of follow-up studies. Psychological Medicine, [s. I.], v. 36, n. 2, p. 159-65, Feb. 2006. Disponível em: https://pubmed.ncbi.nlm.nih.gov/16420712/. Acesso em: 19 jun. 2020.

FAYYAD, J. et al. Cross-national prevalence and correlates of adult attention-deficit hyperactivity disorder. British Journal of Psychiatry, [s. I.], v. 190, n. 5, p. 402-9, June, 2007. Disponível em: https://www. researchgate.net/publication/6359051_Cross-national_prevalence_ and_correlates_of_adult_attention-deficit_hyperactivity_disorder_ Electronic_version. Acesso em: 18 jun. 2020.

FELDMAN, H. M.; REIFF, M. I. Clinical practice. Attention deficithyperactivity disorder in children and adolescents. The New England Journal of Medicine, [s. I.], v. 370, n. 9, p. 838-46, Feb. 2014. Disponível em: https://www.nejm.org/doi/10.1056/NEJMcp1307215. Acesso em: 02 nov. 2020.

FERREIRA ROCHA, M. Déficit de atenção e hiperatividade (TDAH) em sujeito com história de alcoolismo. Renefara, Goiânia, v. 2, n. 2, p. 387411, abr. 2012. Disponível em: http://www.fara.edu.br/sipe/index.php/ renefara/article/view/76. Acesso em: 12 nov. 2020.

FINDLING, R. L. Evolution of the treatment of attention-deficit/ hyperactivity disorder in children: a review. Clinical Theraoeutics, [s. I.], v. 30, n. 5, p. 942-57, May, 2008. Disponível em: https://www. sciencedirect.com/science/article/abs/pii/\$0149291808001768. Acesso em: 08 ago 2020.

FRANKE. B.; NEALE, B. M.; FARAONE, S. V. Genome-wide association studies in ADHD. Human Genettics, [s. I.], n. 126, p. 13-50, 2009. Disponível em: https://link.springer.com/article/10.1007\%2 Fs00439-009-0663-4. Acesso em: 02 nov. 2020.

FRIED, R. et al. Characterizing impaired driving in adults with attentiondeficit/hyperactivity disorder: a controlled study. Journal of Clinical Psychiatry, [s. I.], v. 67, n. 4, p. 567-574, Apr. 2006. Disponível em: https://pubmed.ncbi.nlm.nih.gov/16669721/. Acesso em: 30 jul. 2020.

GARCIA, C. R. et al. The burdened life of adults with ADHD: Impairment beyond comorbidity. European Psychiatry, [s. I.], v. 27, n. 5, p. 309-313, 2012. Disponível em: https://www.sciencedirect.com/science/article/ abs/pii/S0924933810001586. Acesso em: 30 jul. 2020.

GOTO, T. et al. Efficacy and safety of atomoxetine hydrochloride in Asian adults with ADHD: A multinational 10-week randomized double-blind placebo-controlled Asian study. Journal of Attention Disorders, [s. I.], v. 21, n. 2, p. 100-109, Jan. 2017.

HECHTMAN, L. T. Families of children with attention deficit hyperactivity disorder: a review. Canadian Journal of Psychiatry, [s. I.], n. 41, p. 350-60, 1996. Disponível em: https://journals.sagepub.com/doi/ abs/10.1177/070674379604100605. Acesso em: 8 ago 2020.

KESSLER, R. C. et al. The World Health Organization Adult ADHD Self-Report Scale (ASRS): a short screening scale for use in the general population. Psychological Medicine, [s..I], v. 35, n. 2, p. 245-56, Feb. 2005. Disponível em: https://www.researchgate.net/ publication/7895200_The_World_Health_Organization_Adult_ADHD_ Self-Report_Scale_ASRS_a_short_screening_scale_for_use_in_the 
general_population/link/0fcfd512f87fa51f0c000000/download. Acesso em: 13 nov. 2020.

KESSLER, R. C. et al. Structure and diagnosis of adult attention-deficit/ hyperactivity disorder: analysis of expanded symptom criteria from the Adult ADHD Clinical Diagnostic Scale. Arch Gen Psychiatry, [s. I.], v. 67, n. 11, p. 1168-78, Nov. 2010. Disponível em: https://jamanetwork.com/ journals/jamapsychiatry/fullarticle/210916. Acesso em: 08 ago. 2020.

KOOIJ, S. J. et al. European consensus statement on diagnosis and treatment of adult ADHD: The European Network Adult ADHD. BMC Psychiatry, [s. I.], v. 10, n. 1, p. 67, Sept. 2010. Disponível em: https:// link.springer.com/article/10.1186/1471-244X-10-67. Acesso em: 19 ago. 2020.

LEIBSON, C. L. et al. Emergency department use and costs for youth with attention-deficit/hyperactivity disorder: associations with stimulant treatment. Ambulatory Pediatrics, [s. I.], v. 6, n. 1, p. 45-53, 2006. Disponível em: https://www.researchgate.net/publication/7330904_ Emergency_Department_Use_and_Costs_for_Youth_With_AttentionDeficitHyperactivity_Disorder_Associations_With_Stimulant_ Treatment. Acesso em: 31 jul. 2020.

LUNDERVOLD, A. J. et al. Attention Network Test in adults with ADHD - the impact of affective fluctuations. Behavioral and Brain Functions, [s. I.], v. 7, n. 27, p. 1-8, July 2011. Disponível em: https://www. researchgate.net/publication/51525790_Attention_Network_Test_in_ adults_with_ADHD_-_the_impact_of_affective_fluctuations. Acesso em: 19 ago. 2020.

MANNUZZA, S. et al. Impact of the impairment criterion in the diagnosis of adult ADHD: 33-year follow-up study of boys with ADHD. Journal of Attention Disorders, [s. I.], v. 15, n. 2, p. 122-129, 2011. Disponível em: https://www.researchgate.net/publication/43080244_Impact_ of the Impairment_Criterion_in the Diagnosis_of_Adult_ADHD_33Year_Follow-Up_Study_of_Boys_with_ADHD. Acesso em: 08 ago 2020.

MATTOS, P. et al. A multicenter, open-label trial to evaluate the quality of life in adults with ADHD treated with long-acting methylphenidate (OROS MPH): Concerta Quality of Life (CONQoL) study. Journal of Attention Disorders, [s. I.], v. 17, n. 5, p. 444-448, July, 2013. Disponível em: https://journals.sagepub.com/doi/10.1177/1087054711434772. Acesso em: 19 ago. 2020.

MCGOUGH, J. J. et al. Psychiatric comorbidity in adult attention deficit hyperactivity disorder: findings from multiplex families. American Journal of Psychiatry, [s. I.], v. 162, n. 9, p. 1621-7, Sep. 2005. Disponível em: https://ajp.psychiatryonline.org/doi/full/10.1176/appi. ajp.162.9.1621. Acesso em: 31 jul. 2020.

MITCHELL J. T. et al. A pilot trial of mindfulness meditation training for ADHD in adulthood: impact on core symptoms, executive functioning, and emotion dysregulation. Journal of Attention Disorders, [s. I.], v. 21, n. 13, p. 1105-1120, Nov. 2017. Disponível em: https://www.ncbi.nlm. nih.gov/pmc/articles/PMC4045650/. Acesso em: 19 ago. 2020.

OKIE, S. ADHD in adults. New England Journal of Medicine, v. 354, n. 25, p. 2637-2641, June, 2006. Disponível em: http://www.logocom.be/ technisch/medisch/rilatine/pdf/060622-New-England-Journal-ADHD-inadults.pdf. Acesso em: 06 jun. 2020.

PEDUZZI, P. et al. A simulation study of the number of events per variable in logistic regression analysis. J. Clin. Epidemiol., [s.I], v. 49, n. 12, p. 1373-9, Dec 1996. Disponível em: https://pubmed.ncbi.nlm.nih. gov/8970487/. Acesso: 06 jun. 2020.

RAMIREZ, C. A. et al. Anger and anger expression in adults with high ADHD symptoms. Journal of Attentional Disorders, v. 2, n. 2, p. 115-128, 1997. Disponível em: https://psycnet.apa.org/record/1999-00386-004. Acesso em: 21 set. 2020

SABUNCUOGLU, O.; TASER, H.; BERKEM, M. Relationship between traumatic dental injuries and attention-deficit/hyperactivity disorder in children and adolescents: proposal of an explanatory model. Dental Traumatology, [s. I.], v. 21, n. 5, p. 249-253, 2005. Disponível em: https:// www.researchgate.net/publication/7612039_Relationship_between_ traumatic_dental_injuries_and_attention-deficit_hyperactivity_ disorder_in_children_and_adolescents_Proposal_of_an_explanatory model. Acesso em: 10 jul. 2020.

SABUNCUOGLU, O. Traumatic dental injuries and attention-deficit/ hyperactivity disorder: is there a link? Dental Traumatology, [s. I.], v. 23, p. 137-142, 2007. Disponível em: https://pubmed.ncbi.nlm.nih. gov/17511834/. Acesso em: 12 ago. 2020.

SHEEHAN, D. V. et al. The Mini-International Neuropsychiatric Interview (M.I.N.I.): the development and validation of a structured diagnostic psychiatric interview for DSM-IV and ICD-10. J Clin Psychiatry, [s.I], v. 59 Suppl 20, p. 22-33;quiz 34-57, 1998. Disponível em: https://pubmed. ncbi.nlm.nih.gov/9881538/. Acesso em: 08 ago 2020.

SHILON, Y. et al. Accidental injuries are more common in children with attention deficit hyperactivity disorder compared with their nonaffected siblings. Child: Care, Health and Development,[s.I], v. 38, n. 3, p. 366-370, May, 2012. Disponível em: https://pubmed.ncbi.nlm.nih. gov/21722159/. Acesso em: 12 ago. 2020.

SIMON, V. et al. Prevalence and correlates of adult attention-deficit hyperactivity disorder: meta-analysis. British Journal of Psychiatry, [s. I.], v. 194, n. 3, p. 204-11, Mar. 2009. Disponível em: https://www. cambridge.org/core/journals/the-british-journal-of-psychiatry/article/ prevalence-and-correlates-of-adult-attentiondeficit-hyperactivitydisorder-metaanalysis/FBBDADEA596D69D26F49318ECAD410C4. Acesso em: 08 ago 2020.

SPITZER, R. L. et al. DSM-IV casebook: a learning companion to the diagnostic and statistical manual of mental disorders. 4th ed. Washington, DC: American Psychiatric Association, 1994.

SPITZER, R. L.; MD, K. K.; WILLIAMS, J. B. W. Diagnostic and statistical manual of mental disorders. 3th ed. Washington, DC: American Psychiatric Association, 1980. Disponível em: http://citeseerx.ist.psu. edu/viewdoc/summary?doi=10.1.1.459.6032. Acesso em: 8 ago 2020.

STERN, A. et al. The relationship be tween executive functions and quality of life in adults with ADHD. Journal of Attention Disorders, [s. I.], v. 21, n. 4, p. 323-330, 2017. Disponível em: https://journals.sagepub. com/doi/abs/10.1177/1087054713504133. Acesso em: Acesso: 06 jun. 2020.

SOLANTO, M. V. et al. Development of a new psychosocial treatment for adult ADHD. Journal of Attention Disorders, [s. I.], v. 11, n. 6, p. 728-736, 2008. Disponível em: https://journals.sagepub.com/doi/ abs/10.1177/1087054707305100. Acesso em: Acesso: 06 jun. 2020.

TESTA, M. A.; SIMONSON, D. C. Assesment of quality-of-life outcomes. The New England of Journal of Medicine, v. 334, n. 13, p. 835-840, Mar. 1996. Disponível em: https://www.nejm.org/doi/full/10.1056/ NEJM199603283341306. Acesso em: 17 set. 2020.

THIKKURISSY, S.; McTIGUE, D. J.; COURY, D. L. Children presenting with dental trauma are more hyperactive than controls as measured by the ADHD rating scale IV. Pediatr Dent., [s. I.], v. 34, n. 1, p. 28-31, 2012. Disponível em: https://pubmed.ncbi.nlm.nih.gov/22353453/. Acesso em: 14 nov. 2020.

TODD, R. D. Genetics of attention déficit/hyperactivity disorder: are we ready for molecular genetic studies? American Journal of Medical Genenetics, [s. I.], v. 96, n. 3, p. 241-243, 2000. Disponivel em: https://onlinelibrary.wiley.com/doi/abs/10.1002/10968628(20000612)96:3\%3C241::AID-AJMG1\%3E3.0.CO;2-R. Acesso em: 15 nov. 2020.

TOSHINOBU, T. et al. Parental ADHD status and its association with 
Proband ADHD subtype and severity. Journal of Pediatrics, [s. I.], v. 157, n. 6, p. 995-1000, 2010. Disponível em: https://www.sciencedirect.com/ science/article/abs/pii/S0022347610004804. Acesso em: 12 out. 2020.

TSUJII, N. et al. Effect of continuing and discontinuing medications on quality of life after symptomatic remission in Attention-Deficit/ Hyperactivity Disorder: a aystematic review and meta-analysis. Journal of Clinical Psychiatry, [s. I.], v. 81, n. 3, 2020. Disponível em: https://pesquisa.bvsalud.org/controlecancer/resource/pt/mdl32237294 ?src=similardocs. Acesso em: 19 ago. 2020.

VADALÀ, R. et al. Attention deficit hyperactivity disorder (ADHD): from a childhood neuropsychiatric disorder to an adult condition. Functional Neurology, [s. I.], v. 26, n. 3, p. 117-119, Jul.-Sep. 2011. Disponível em: https://www.ncbi.nlm.nih.gov/pmc/articles/PMC3814555/. Acesso em: 31 jul. 2020.

VOLKOW, N. D.; SWANSON, J. M. Clinical practice: adult attention deficithyperactivity disorder. The New England Journal of Medicine, v. 369, n. 20, p. 1935-1944, Nov. 2013. Disponível em: https://escholarship. org/uc/item/6mt6h1zn. Acesso em: 29 ago. 2020.

WHALEN, C. K. et al. The ADHD spectrum and everyday life: experience sampling of adolescent moods, activities, smoking and drinking. Child Development, [s. I.], v. 73, n. 1, p. 209-227, Jan.-Feb. 2002. Disponível em: https://www.jstor.org/stable/3696440?seq=1. Acesso em: 19 ago. 2020.

WEHMEIER, P. M. et al. Effect of atomoxetine on quality of life and family burden: results from a randomized, placebo-controlled, double-blind study in children and adolescents with ADHD and comorbid oppositional defiant or conduct disorder. Quality of Life Research, [s. I.], v. 20, n. 5, p. 691-702, June, 2011. Disponivel em: https://www.jstor.org/ stable/41488124?seq=1. Acesso em: 06 jun. 2020.

WILENS, T. E. et al. Correlates of alcohol use in adults with ADHD and comorbid alcohol use disorders: exploratory analysis of a placebocontrolled trial of atomoxetine. Current Medical and Research and Opinion, [s. I.]. v. 27, n. 12, p. 2309-20, Dec. 2011. Disponível em: https://pubmed.ncbi.nlm.nih.gov/22029549/. Acesso em: 19 ago. 2020.

Submetido em: 08/12/2020

Aceito em: 14/12/2020 